TA04 11:20

\title{
Robust Performance Analysis for a Class of Uncertain Nonlinear Systems
}

\author{
Jorge E. Tierno* \\ Electrical Engineering, 116-81 \\ California Institute of Technology \\ Pasadena, CA 91125, U.S.A.
}

\author{
Richard M. Murray \\ Mechanical Engineering, 104-44 \\ California Institute of Technology \\ Pasadena, CA 91125, U.S.A.
}

\begin{abstract}
Performance analysis of a large class of nonlinear systems is proven to be equivalent to performance analysis of a constrained uncertain linear system, for which computable analysis methods have already been developed.
\end{abstract}

Keywords: robustness analysis, nonlinear systems, computational complexity.

\section{Introduction}

Theoretical and computational tools for analysis and synthesis of robust controllers for linear systems are well developed in a variety of instances. Controllers generated with these tools can provide guaranteed performance in the presence of structured uncertainty, and the worst case disturbances for a given controller can be determined. A recent description of this approach can be found in [4].

For linear time invariant (LTI) systems with complex, structured uncertainty, analysis of robust performance can be reduced to searching for the solution of a set of algebraic equations which give bounds on the achievable performance. One is thus able to find computationally effcient solutions, such as the power algorithm for the $\mu$ lower bound, without doing an explicit parameter search involving repeated simulation. This works because the system is linear and the performance and uncertainty descriptions are chosen so as to give computationally attractive solutions, even for large problems.

Current research in linear systems theory is devoted to extending the existing theory to incorporate more realistic uncertainty structures (such as real parameters) and to solving linear time varying problems.

Analysis of nonlinear systems on the other hand has stayed mainly at the theoretical level. Lower bounds are usually computed through extensive simulation or local optimization techniques. However these methods require large amounts of computation; standard optimization techniques fail even for small problems, and a search over parameter space exhibits exponential growth with the number of parameters. Several upper bounds for performance of nonlinear systems have been developed in the last few years. Most of these results are generalizations of Lyapunov theorems and depend on us being able to find a Lyapunov function for the system. However, there are no systematic ways to accomplish this.

The success of practical methods for analysis of linear systems, can be explained to some extent by the fact that

\footnotetext{
*E-Mail: jorgenot.caltech.edu
}

linear systems can be naturally described by finite amounts of data, and that therefore, properties of the system can be expressed as functions on a finite dimensional space. If we want to replicate that success for nonlinear systems we have to restrict our search to classes of nonlinear systems that can be described finitely.

In [6] we showed how by considering systems over a finite time horizon, an efficient algorithm for a lower bound on the robust performance level could be developed. Since lower bounds require only local information, the finite description of a system is in this case simply a simulation process: i.e. a computer program that returns the outputs corresponding to a given input; finite time horizon is required to guarantee finite simulation time.

For the upper bound case, as opposed to the lower bound, since we are looking for global instead of local results we need to be able to describe the system globally with finite data. We present in this paper a large class of problems that accept such a representation. We will also show that for problems in that class an important measure of performance can be evaluated by analyzing an auxiliary linear system constructed from the original nonlinear one.

The linear problem belongs to a class for which analysis methods have already been developed. In particular an upper bound on the performance of the linear problem will also be an upper bound for the performance of the original nonlinear one. Together with the lower bound developed in [6], these two results extend the robustness analysis tools available for linear systems, to the nonlinear case.

The organization of this paper is as follows: in Section 2 we describe the robust trajectory tracking problem and the class of nonlinear systems for which we will solve it; in Section 3 we will build the auxiliary linear system and prove technical lemmas that connect the behavior of the two; finally in Section 4 we prove the main result of this paper establishing the equivalence in the behavior of the nonlinear and auxiliary system, and we derive sufficient conditions for performance.

\section{Nonlinear Robust Performance}

\subsection{The robust trajectory tracking problem}

Many nonlinear analysis problems of engineering interest can be reduced to the problem of tracking a nominal tra jectory. Be it a car changing lanes on an automated highway, an airplane performing a turn, or an idling engine going through a sudden change in load, the designer has in mind an appropriate path, to be completed in a finite predetermined time, and builds his control system accordingly. Since the real system is not exactly the one used for the design, and it is also subject to noise, the system 
will not follow the intended trajectory. However, the design can still be considered successful if the real trajectory remains close enough to the nominal or expected one in an appropriate norm.

In this paper we will consider a restricted version of this problem. Our performance measure will be the 2-norm of the error signal (i.e. the difference between the nominal and the actual trajectory.) If needed the error signal can be weighted by a multiplicative time function. Noise signals will be bounded in the 2-norm. The system equations will be allowed to depend on a set of real parameters varying in closed intervals. The initial conditions for some or all of the state variables will also be allowed to vary in given closed intervals.

To simplify the notation we will work in the following with a system with one uncertain parameter and one noisy input. However all the results presented generalize naturally.

Let $u$ be the noise signal perturbing the system, and let the error signal $y$ be the difference between the nominal and the actual trajectories. The equations describing the system will then be:

$$
\begin{aligned}
& x_{k+1}=f\left(x_{k}, u_{k}, \delta, k\right) \\
& y_{k}=g\left(x_{k}, u_{k}, \delta, k\right)
\end{aligned}
$$

with the following constraints:

$$
\begin{aligned}
|\delta| & \leq 1 \\
\|u\|_{2} & \leq 1
\end{aligned}
$$

For a given level of performance $\gamma$ the robust performance question can be set as a feasibility question. The performance level $\gamma$ is met if and only if the following set of equations:

$$
\begin{aligned}
& x_{k+1}=f\left(x_{k}, u_{k}, \delta, k\right) \\
& y_{k}=g\left(x_{k}, u_{k}, \delta, k\right)
\end{aligned}
$$

with the following constraints:

$$
\begin{aligned}
|\delta| & \leq 1 \\
\|u\|_{2} & \leq 1 \\
\|y\|_{2} & \geq \gamma
\end{aligned}
$$

admits no solutions.

\subsection{Finite global descriptions}

Our goal is to develop algorithms, suitable for implementation on a digital computer, to compute a global upper bound for the performance of a nonlinear system. A fundamental precondition for the development of such an algorithm is a finite description of the nonlinear system. Note that a finite description implies more than specifying the problem with finitely many characters; the decoding of such a description has to be doable by a finite procedure as well. Given the large variety of behaviors in nonlinear systems, it is doubtful that such an encoding exists for a generality of problems. However, we will exhibit a large class of nonlinear problems, that we believe to be of engineering relevance, for which we can develop a finite representation and derive from it efficient analysis algorithms.

\section{The class of rational nonlinear systems}

The class of problems we will study consists of discrete time nonlinear systems, with a finite time horizon performance specification. (That is to say we are only concerned with the behavior of the system over the first $T$ time steps.) The evolution of the state $x$ and output $y$ of the system will be governed by the equations:

$$
\begin{aligned}
& x_{k+1}=f\left(x_{k}, u_{k}, \delta^{p}, k\right) \\
& y_{k}=g\left(x_{k}, u_{k}, \delta^{p}, k\right) \quad k=1, \ldots, T
\end{aligned}
$$

where $u$ is the vector of input signals, $\delta^{p}$ is a vector of uncertain parameters, and $x_{1}$ is the initial state. Furthermore, we will require the functions $f$ and $g$ to be rational expressions of the state, input and parameter variables. We will also require the following well posedeness condition: there exist vectors of positive constants $K_{k}^{x}, K_{k}^{u}$, and $K_{k}^{\delta}$ such that $f\left(x_{k}, u_{k}, \delta^{p}, k\right)$ and $g\left(x_{k}, u_{k}, \delta^{p}, k\right)$ are well defined for all $x, u$, and $\delta^{p}$ satisfying:

$$
\left|x_{k}\right|<K_{k}^{x}, \quad\left|u_{k}\right|<K_{k}^{u}, \quad\left|\delta_{k}^{p}\right|<K_{k}^{\delta}
$$

where absolute values and inequalities are meant in the element by element sense. Furthermore we will require that if the input signals and parameters remain within those given bounds for all $k=1, \ldots, T$, and the initial state $x_{1}$ verifies $\left|x_{1}\right|<K_{1}^{x}$, then the state remains within its bounds for all $k=1, \ldots, T$. Finally we will assume that 0 is an equilibrium point, i.e. $f\left(0,0, \delta^{p}, k\right)=f\left(0,0, \delta^{p}, k\right)=0$.

\section{Constant matrix representation}

In this section we will derive a constant matrix representation for a system in the class defined previously. To simplify the notation we will consider a system with one state, one scalar input and one uncertain parameter. The extension to a general problem in the class is straightforward.

As $f$ and $g$ are rational functions of $x_{k}$ and $u_{k}$ that do not blow up at zero, they can be expressed as linear fractional transformations on those same variables. This means that there exists a matrix $M_{k}$ whose entries only depend on $k$ and natural numbers $m_{x}$ and $m_{u}$ such that

$$
\left[\begin{array}{c}
f\left(x_{k}, u_{k}, \delta_{k}^{p}, k\right) \\
g\left(x_{k}, u_{k}, \delta_{k}^{p}, k\right)
\end{array}\right]=M_{k} * D,
$$

where $*$ denotes the Redheaffer star-product, and $D$ is the diagonal matrix:

$$
D=\operatorname{diag}\left(\delta_{k}^{p}, x_{k} I_{m_{x}}, u_{k} I_{m_{u}}\right) .
$$

\section{Auxiliary Linear System Construction}

In this section we will construct a linear system starting from the matrix $M$ of the finite representation of the nonlinear system discussed in the previous section. We will do this construction in two stages. First for a one time step system, and then for a $T$ time step system. Since the resulting linear system will be in implicit form, we include also a section reviewing the definition and some recent results on this kind of systems.

\subsection{Implicit uncertain linear systems}

Recent developments in linear system analysis have shown that it is interesting to answer the following question: Given two matrices $A$ and $C$ and a block diagonal uncertainty structure $\Delta$, does the system of equations:

$$
\begin{aligned}
z & =A w \\
w & =\Delta z \\
0 & =C w
\end{aligned}
$$




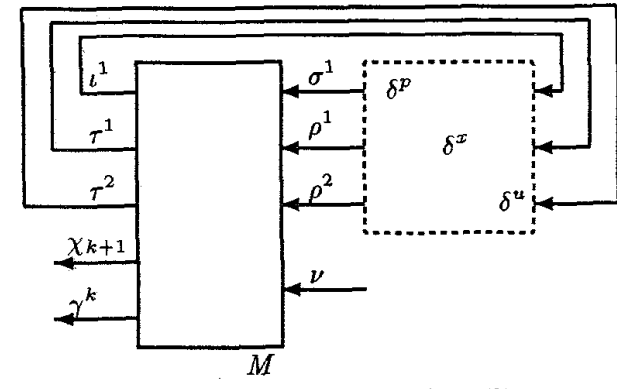

Figure 1: First step in construction of auxiliary system

admit more than one solution for some allowable value of the uncertain matrix $\Delta$ ? A complete description of this problem is given in [5], where it is also shown that this question can be answered by computing a quantity $\mu_{\Delta}(A, C)$, similar in nature to the structured singular value. It is also shown there that upper and lower bounds similar in nature to those for the standard structured singular value can be developed to compute that quantity. We will need in this paper a sufficient condition for the answer to the question to be no. We reproduce here without proof the following:

Theorem 1 [5] If there is a solution to the linear matrix inequality:

$$
C^{\perp}\left(A^{t} D_{l}^{*} D_{l} A-D_{r}^{*} D_{r}\right) C^{\perp^{*}}<0
$$

where $D_{r}$ and $D_{l}$ are positive definite matrices verifying $D_{l} \Delta=\Delta D_{r}$ for all $\Delta$ with $\|\Delta\| \leq 1$, then the system of equations (9) admits only the trivial solution.

In general an implicit uncertain system, denoted $(A, C, \Delta)$ with input $u$ and output $y$ is defined by the system of equations

$$
\left[\begin{array}{l}
z \\
y
\end{array}\right]=A\left[\begin{array}{l}
v \\
u
\end{array}\right]
$$$$
v=\Delta z \text {. }
$$

\subsection{Auxiliary Linear System}

We will construct an auxiliary linear system from the original nonlinear one through a series of steps.

\section{Auxiliary system for a length 1 time horizon}

First, consider the uncertain linear system with matrix $M$ (from the previous section) and uncertainty structure $\Delta=$ $\operatorname{diag}\left(\delta^{p}, \delta^{x} I_{m_{x}}, \delta^{u} I_{m_{u}}\right)$. This system is then governed by the equations

$$
\left[\begin{array}{c}
\chi_{k+1} \\
\gamma_{k}
\end{array}\right]=(M * \Delta) \nu
$$

A block diagram for this system is shown in Figure 1.

Partition the matrix $M$ compatibly with the signals in Figure 1. Extend this linear system to have four extra inputs $v^{1}, v^{2}, \omega^{1}$, and $\omega^{2}$ and two outputs $\zeta^{1}$ and $\zeta^{2}$ and extend also the corresponding uncertainty structure as shown in Figure 2. Denote by $M_{e}$ and $\Delta_{e}$ the resulting system

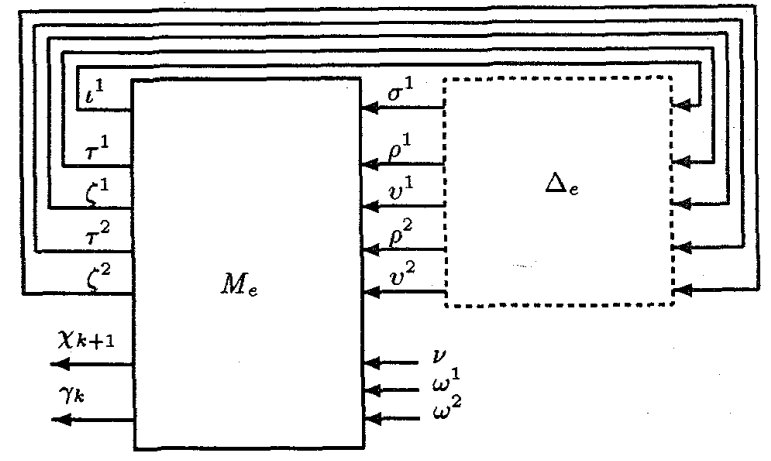

Figure 2: Second step in construction of auxiliary system matrix and uncertainty structure. We will have:

$$
M_{e}=\left[\begin{array}{cccccccc}
M_{11} & M_{12} & 0 & M_{13} & 0 & M_{14} & 0 & 0 \\
M_{21} & M_{22} & 0 & M_{23} & 0 & M_{24} & 0 & 0 \\
0 & 0 & 0 & 0 & 0 & 1 & 0 & 0 \\
M_{31} & M_{32} & 0 & M_{33} & 0 & M_{34} & 0 & 0 \\
0 & 0 & 0 & 0 & 0 & 1 & 0 & 0 \\
M_{41} & M_{42} & 0 & M_{43} & 0 & M_{44} & 0 & 0 \\
M_{51} & M_{52} & 0 & M_{53} & 0 & M_{54} & 0 & 0
\end{array}\right]
$$

and

$$
\Delta_{e}=\left[\begin{array}{llll}
\delta & & & \\
\delta^{x} I & & \\
& \delta^{x} & \\
& & \delta^{u} I \\
& & & \delta^{u}
\end{array}\right] .
$$

The final step in the development of the auxiliary system is adding linear implicit constraint on the inputs signals. Add to the system in Figure 2 the two linear constraints:

$$
\begin{aligned}
& v^{1}-\omega^{1}=0 \\
& v^{2}-\omega^{2}=0 .
\end{aligned}
$$

The resulting set of equations characterizes an implicit uncertain linear system as those described in Section 3.1, and can be represented by the block diagram in Figure 3, where $C_{e}$ is the matrix

$$
C_{e}=\left[\begin{array}{cccccccc}
0 & 0 & 1 & 0 & 0 & 0 & -1 & 0 \\
0 & 0 & 0 & 0 & 1 & 0 & 0 & -1
\end{array}\right] .
$$

The following lemma establishes the conection between the nonlinear and the auxiliary system.

Lemma 1 If $\nu=1, \omega^{1}=x_{k}$, and $\omega^{2}=u_{k}$ then

$$
\begin{aligned}
& \chi_{k+1}=f\left(x_{k}, u_{k}, \delta_{k}^{p}, k\right)=x_{k+1} \\
& \gamma_{k} \quad=g\left(x_{k}, u_{k}, \delta_{k}^{p}, k\right)=y_{k}
\end{aligned}
$$

Proof: Note that the extension of the system was done so that

$$
\begin{aligned}
& v^{1}=\delta^{x} \nu \\
& v^{2}=\delta^{u} \nu .
\end{aligned}
$$

So, setting $\nu=1$ in equations (19), we have $v^{i}=\delta^{i}$. Substituting these expressions in equations (16) we have

$$
\begin{aligned}
& \delta^{x}=\omega^{1}=x_{k} \\
& \delta^{u}=\omega^{2}=u_{k} .
\end{aligned}
$$




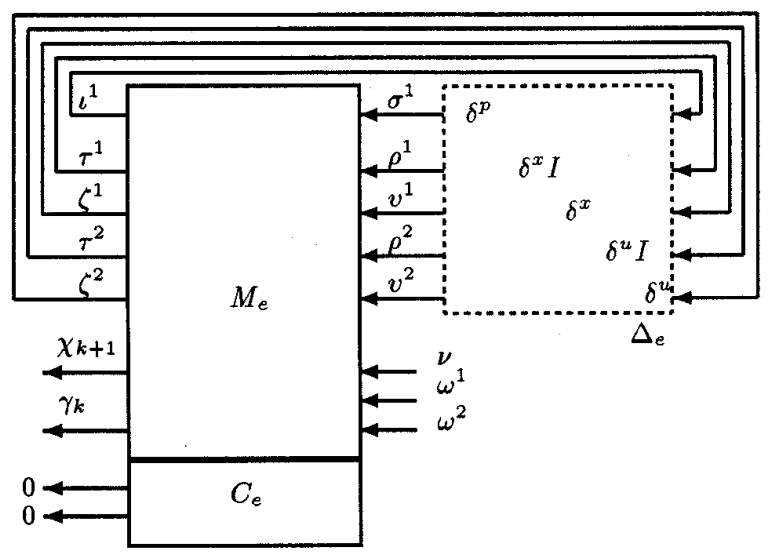

Figure 3: Third step in construction of auxiliary system

Since

$$
\begin{aligned}
& {\left[\begin{array}{c}
\chi_{k+1} \\
\gamma_{k}
\end{array}\right]=(M * \Delta) \nu} \\
& {\left[\begin{array}{c}
x_{k+1} \\
y_{k}
\end{array}\right]=M * D,}
\end{aligned}
$$

substitution of (20) in (21) gives the desired result.

We finish this section with a technical lemma we will need for the main theorem of this paper:

Lemma 2 If $\nu=\omega^{1}=\omega^{2}=0, \delta^{p}<K^{p},\left|\delta^{x}\right|<K^{x}$, and $\left|\delta^{u}\right|<K^{u}$ then all signals in Figure 3 are 0 .

Proof: The norm bound conditions in $\delta^{p}, \delta^{x}$ and $\delta^{u}$, imply that $M_{e} * \Delta_{e}$ is a well posed linear fractional transformation. This means that the system of linear equations it defines has a unique solution. Since for a linear system $\overrightarrow{0}$ is a solution when the inputs are set to 0 the lemma follows.

\section{Auxiliary system for a length $T$ time horizon}

For one time step, Lemma 1 shows that the auxiliary linear system state and outputs track those of the nonlinear one. This result can be extended to any time horizon of finite length $T$ by concatenating as many instances of the auxiliary system in a simple fashion. In this section we show how to carry out that concatenation. For simplicity in notation we will show how to do this concatenation for two time steps; the generalization to any finite number of steps is straightforward. Consider the matrices $M_{e}^{1}, M_{e}^{2}, \Delta_{e}^{1}$, and $\Delta_{e}^{2}$, that define the auxiliary system for the first two time steps. First, connect them as shown in Figure 4, to form another uncertain system denoted $\left(M_{c}, \Delta_{c}\right)$ The input vector $a$ to the matrix $M_{c}$ and the corresponding output vector will inherit from the auxiliary systems at time steps 1 and 2 the following partition:

$$
\begin{aligned}
& a=\left[\iota_{1}^{1}, \rho_{1}^{1}, v_{1}^{1}, \rho_{1}^{2}, v_{1}^{2}, \iota_{2}^{1}, \rho_{2}^{1}, v_{2}^{1}, \rho_{2}^{2}, v_{2}^{2}, \omega_{1}^{1}, \omega_{1}^{2}, \omega_{2}^{2}, \nu\right]^{t} \\
& b=\left[\sigma_{1}^{1}, \tau_{1}^{1}, \zeta_{1}^{1}, \tau_{1}^{2}, \zeta_{1}^{2}, \sigma_{2}^{1}, \tau_{2}^{1}, \zeta_{2}^{1}, \tau_{2}^{2}, \zeta_{2}^{2}, \chi_{3}, \gamma_{1}, \gamma_{2}\right]^{t} .
\end{aligned}
$$

From Figure 4 note that the input $\omega^{1}$ of the second auxiliary system has been connected to a signal whose value is

$$
\omega_{2}^{1}=\left[M_{31}(1) 0 M_{32}(1) 00000000 M_{33}(1)\right] a .
$$

where $M_{i j}(1)$ corresponds to the $i, j$ element of the partition of the matrix for the first auxiliary system used in previous

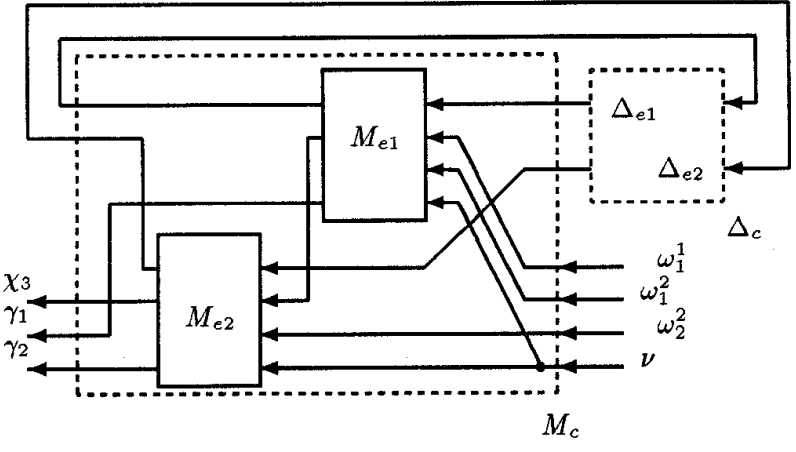

Figure 4: Two time-steps auxiliary system

section, and the 0 entries have dimensions compatible with the given partition of $a$. Add then to the system $\left(M_{c}, \Delta\right)$ the following constraints in $a$ :

$$
\begin{aligned}
v_{1}^{1}-w_{1}^{1} & =0 \\
v_{1}^{2}-w_{1}^{2} & =0 \\
v_{2}^{1}-\left[M_{31}(1) 0 M_{32}(1) 00000000 M_{33}(1)\right] a & =0 \\
v_{2}^{2}-w_{2}^{2} & =0
\end{aligned}
$$

We can write all this constraints as one vectorial equation in $a$ :

$$
C a=0 .
$$

We have thus defined a new implicit uncertain system $\left(M_{c}, C, \Delta_{e}\right)$. The following is a direct consequence of Lemma 1.

Lemma 3 If $\nu=1, \omega_{1}^{1}=x_{1}, \omega_{1}^{2}=u_{1}$, and $\omega_{1}^{2}=u_{2}$ then

$$
\begin{aligned}
& \chi_{3}=x_{3} \\
& \gamma_{k}=y_{k} \quad k=1,2
\end{aligned}
$$

Proof: It follows from applying Lemma 1 to the first component of $M_{c}$, concluding that the $\omega^{2}$ input of the second half is connected to a signal of value $x_{2}$, and applying again Lemma 1 to this second half.

We can repeat this procedure for all time steps. Note that in the final step we can include or omit the final state as part of the output. Whether we do so or not will depend on the nature of our problem and the performance specification being considered.

The second lemma of the preceeding section also generalizes to the length $T$ horizon:

Lemma 4 If $\nu=\omega_{1}^{1}=\omega_{k}^{2}=0$, for $k=1,2,\left|\delta_{k}^{p}\right|<K_{k}^{p}$, $\left|\delta_{k}^{x}\right|<K_{k}^{x}$, and $\left|\delta_{k}^{u}\right|<K_{k}^{u}$ then all signals in Figure 4 are 0 .

Proof: Apply Lemma 2 to the first block in the interconnection. Note that all signals that are propagated to the next block are zero, and apply Lemma 2 again.

Once again the generalization of this lemma to $T$ time steps is immediate.

\section{Performance Analysis}

We will now proceed to show how the auxiliary system just defined can be used to analyze performance for the class of nonlinear systems discussed in Section 2.2.

\subsection{Nonlinear and auxiliary performance}

In order to be able to convert the performance specification 


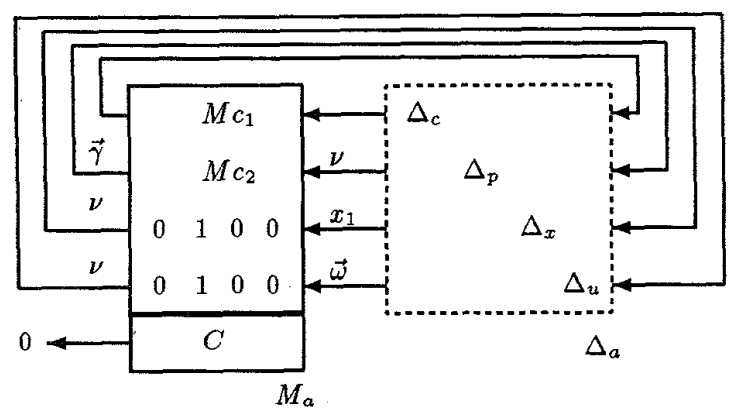

Figure 5: Analysis setup

one we will have to modify the latter slightly. Add one more output to the system $\left(M_{c}, C, \Delta\right)$ by modifying the matrix $M_{c}$ as shown in Figure 5. The constrained linear uncertain system in Figure 5 will be denoted $\left(M_{a}, C, \Delta_{a}\right)$.

Define the "norm bounded" uncertainty structures:

$$
\begin{array}{r}
\mathbf{B} \Delta_{\mathbf{e}}=\left\{\text { blockdiag } \left(\delta_{1}^{p}, \delta_{1}^{x} I_{m_{x}+1}, \delta_{1}^{u} I_{m_{u}+1}, \delta_{2}^{p}, \delta_{2}^{x} I_{m_{x}+1}\right.\right. \\
\left.\delta_{2}^{u} I_{m_{u}+1}, \ldots, \delta_{T}^{p}, \delta_{T}^{x} I_{m_{x}+1}, \delta_{T}^{u} I_{m_{x}+1}\right), \\
\left.\left|\delta_{i}^{p}\right| \leq K_{i}^{p},\left|\delta_{i}^{x}\right| \leq K_{i}^{u} i=1, \ldots, T\right\}
\end{array}
$$

and

$$
\begin{gathered}
\mathbf{B} \Delta_{a}=\left\{\operatorname{blockdiag}\left(\Delta_{e}, \Delta_{p}, \Delta_{x_{1}}, \Delta_{\omega}\right), \Delta_{e} \in \mathbf{B} \Delta,\right. \\
\left.\left\|\Delta_{p}\right\| \leq 1,\left\|\Delta_{x_{1}}\right\| \leq 1,\left\|\Delta_{\omega}\right\| \leq 1\right\}
\end{gathered}
$$

where $\Delta_{p}, \Delta_{x_{1}}$, and $\Delta_{\omega}$ are full matrices with dimensions defined by Figure 5. (See [7] for a more detailed study of this analysis setup.)

We are now ready to state the main theorem of this paper that will allow us to derive a sufficient condition for performance of the original nonlinear system.

Theorem 2 For all signals $u$ with $\|u\|_{2} \leq 1$ and $\left|u_{k}\right|<K_{k}^{u}$ and all initial states $x_{1}<K_{k}^{u}$ we will have $\|y\|_{2} \leq 1$ if and only if the performance auxiliary system is well posed for all $\Delta_{a} \in \mathbf{B} \boldsymbol{\Delta}_{\mathbf{a}}$

Proof: First assume the system has a nonzero solution. Then $\nu \neq 0$ because otherwise all signals are 0 . This follows from $\vec{\omega}=\Delta_{\omega} \nu, x_{1}=\Delta_{x} \nu$ and lemma 4 . Since the equations are linear then there is also a solution with $\nu=1$. The condition $\left\|\Delta_{u}\right\| \leq 1$ implies that $\|\vec{\omega}\| \leq 1$, and the condition $\left\|\Delta_{p}\right\| \leq 1$ implies then that $\|\vec{\gamma}\| \geq 1$. Since $\nu=1$ from Lemma 3 we conclude that there is an input and initial condition for the nonlinear system within the specified bounds such that $\|y\|>1$, and so the performance requirement is not met.

Conversely assume that an allowable initial state $x_{1}$ and an allowable input signal $u$ exist such that $\|y\| \geq 1$. Then setting $\nu=1, \vec{\omega}=u$, and $\omega_{1}^{1}=x_{1}$ in the set of equations represented by Figure 5, we have $\|\gamma\| \geq 1$, and consequently there exists a $\Delta_{a} \in \mathbf{B} \boldsymbol{\Delta}_{a}$ such that the given system of equations has a nonzero solution.

This theorem establishes the equivalence between analysis of a nonlinear system and analysis of a linear one. The solution of the given linear problem is NP-Complete. The results thus tells us that solving the performance analysis question in the class of nonlinear systems being considered is not worse than an NP-Complete problem whose size grows with the length of the time horizon.

\subsection{Sufficient conditions for performance}

Once the problem of performance of the nonlinear system has been reduced to a performance question over a linear one, we can use the results of the linear theory to analyze problems in our class.

Note that without loss of generality we can assume that the performance bounds, the norm of the input and the bounds $K^{x}$ and $K^{u}$ are all 1. Putting Theorems 2 and 1 together we conclude that:

Theorem 3 The nonlinear system in (5) verifies the given performance specification if there are positive definite matrices $D_{l}$ and $D_{r}$ verifying $\Delta_{a} D_{l}=D_{r} \Delta_{a}$ for all $\Delta_{a} \in \Delta_{a}$ and such that:

$$
C^{\perp}\left(M_{a}^{*} D_{l}^{*} D_{l} M_{a}-D_{r}^{*} D_{r}\right) C^{\perp *}<0
$$

A sufficient condition for performance of a nonlinear system can thus be reduced to solving a convex optimization problem over a finite dimensional space. The number of parameters in the optimization problem grows linearly with the length of the horizon $T$. The optimization problem takes the form of a Linear Matrix Inequality (LMI). Recent developments in systems theory have shown that several important problems can be reduced to solving LMI's and consequently a significant effort has been put in developing practical algorithms for them [3]. Commercial packages are available that implement some of these methods [2].

Current LMI solving algorithms usually have computation time growth proportional to the cube of the size of the matrix $M_{a}$. However these algorithms do not exploit the specific structure of our matrices and it is conceivable that cubic growth with $T$ can be avoided by tailoring the standard algorithms to our specific case.

\section{Example}

We will test the analysis technique on simplified dynamics for the ducted fan experimental setup described in [1]. The nonlinear dynamics used are

$$
\left\{\begin{array}{l}
\dot{x}_{1}=x_{2} \\
\dot{x}_{2}=u_{1}+u_{2} x_{5} \\
\dot{x}_{3}=x_{4} \\
\dot{x}_{4}=-u_{1} x_{5}+u_{2} \\
\dot{x}_{5}=x_{6} \\
\dot{x}_{6}=r u_{1}+k x_{2} x_{5}
\end{array}\right.
$$

with the outputs defined as

$$
\left\{\begin{array}{l}
y_{1}=x_{1} \\
y_{2}=x_{3} \\
y_{3}=x_{5}
\end{array}\right.
$$

Since $x_{5}$ appears in all the nonlinear terms, we will rewrite these equations as

$$
\left\{\begin{array}{l}
\dot{x}_{1}=x_{2} \\
\dot{x}_{2}=u_{1}+u_{2} \delta \\
\dot{x}_{3}=x_{4} \\
\dot{x}_{4}=-u_{1} x_{5}+u_{2} \\
\dot{x}_{5}=x_{6} \\
\dot{x}_{6}=r u_{1}+k x_{2} \delta
\end{array}\right.
$$

with the constraint

$$
\delta-x_{5}=0
$$


We will consider a 1 second time horizon. The 2-norm of the combined inputs will be set at 0.1 and our performance measure will be the 2-norm of the combined outputs. As a first step we compute a lower bound for the performance using the procedure described in [6]. To compute an upper bound we convert the system into a discrete time one, with a sample time of $0.05 \mathrm{~s}$. The auxiliary linear system corresponding to the 20 time samples is represented by a 147 by 127 system matrix and a 20 by 127 constraints matrix. The corresponding LMI has 223 decision variables. The procedure described above answers the question can the norm of the output be bigger than $\gamma$ ? Since we have a lower bound for the maximum $\gamma$ we will ask the question for a succession of values. The results obtained are shown in Table 1 . The computations where made with the Matlab toolbox LMILab. The column labeled $\rho$ (RHS) indicates the maximum eigenvalue achieved in the right hand side of the LMI.

\begin{tabular}{c|c|c|c}
$\gamma / \gamma_{l}$ & Feasible & Iter. & $\rho$ (RHS) \\
\hline 1 & no & & .96 \\
3 & yes & 34 & -496 \\
2 & yes & 117 & -0.8868 \\
1.5 & no & & 0.96 \\
1.75 & no & & 0.96 \\
1.85 & no & & 0.96 \\
1.95 & yes & 160 & -0.452
\end{tabular}

Table 1: Upper bound feasibility

Remarks: The LMI solver stops if after a certain number of iterations it cannot improve the performance index. Thus reports of non feasibility could in principle not be true. This is consistent with the nature of the test. As an upper bound it only guarantees that the error will not be worse than a certain amount. There is a tradeoff between computation time, and the precision with which we would like to have the answer. It is thus of fundamental importance to have both an upper and lower bound.

\section{Conclusion}

The main result of this paper (Theorem 2) proves the equivalence of the questions "Does an uncertain rational nonlinear system always meet a given performance specification?" and the apparently simpler one: "Does a linear uncertain system always meet a given performance specification?"

From this equivalence we derived a convex upper bound for a large class of nonlinear performance problems that takes the form of a finite Linear Matrix Inequality. This result, together with the algorithm for computing a lower bound for the same class of problems presented in [6], provides a very good first step in the direction of extending the tools for analysis of linear systems to nonlinear ones.

The upper bound derived from Theorem 2 is difficult to compute even for a small problem with current technology, since the size of the resulting LMI exceeds the capability of LMI solvers like "LMI-Lab". For example evaluating the performance condition over a 9 second time horizon for the full nonlinear model of the Caltech ducted fan experimental setup [1] will give an LMI with approximately 3000 decision variables. However a large effort is being put into the development of algorithms to solve LMI's and we believe that the technology to compute the nonlinear upper bounds derived in this paper will soon be available. Since the number of parameter grows linearly with the time horizon, a tenfold increase in computer speed will allow us to solve a problem ten times bigger. On the other hand solving the problem in the traditional way by gridding the initial state, noise and parameter spaces in order to obtain a global answer results in problems with exponential growth; consequently these methods will benefit far less from increased computation speed.

Another consequence of Theorem 2 is that robustness analysis of performance for the class of nonlinear systems presented in this paper is proved to be not intrinsically harder than analysis of Linear Systems, from a computational complexity point of view.

\section{Acknowledgements}

The authors are grateful to John C. Doyle for discussions on this paper. This work was partially supported by NASA and AFOSR grant number F 49620-95-1-0419.

\section{References}

[1] H. Choi, P. Sturdza, and R. M. Murray. Design and construction of a small ducted fan engine for nonlinear control experiments. In Proceedings of the American Control Conference, pages 2618-2622, 1994.

[2] P. Gahinet, A. Nemiroskii, A. Laub, and M. Chilali. The LMI Control Toolbox. The MathWorks Inc., 1994.

[3] Y. Nesterov and A. Nemirovskii. Interior-Point Polynomial Algorithms in Convex Programming, volume 13 of Studies in Applied Mathematics. SIAM, 1994.

[4] A. Packard and J. C. Doyle. The complex structured singular value. Automatica, 29(1):71-109, 1993.

[5] F. Paganini and J.C. Doyle. Analysis of implicitly defined systems. In Proceedings of the $33^{\text {rd }}$ Conference on Decision and Control, pages 3673-3678. IEEE, 1994.

[6] J. Tierno, R. Murray, and J.C. Doyle. An efficient algorithm for performance analysis of nonlinear control systems. In Proceedings of the 1995 American Control Conference, pages 2717-2721, 1995.

[7] J.E. Tierno and J.C. Doyle. Finite time horizon robust performance analysis. In Proceedings of the $33^{\text {rd }}$ Conference on Decision and Control, pages 3080-3085. IEEE, 1994. 